\title{
Myriam Kohnen, Mobilis in mobili. Le Corps en mouvement dans la littérature du $\mathrm{XIX}^{\mathrm{e}}$ siècle
}

\section{Maria Emanuela Raffi}

\section{OpenEdition}

\section{Journals}

\section{Edizione digitale}

URL: https://journals.openedition.org/studifrancesi/21526

DOI: 10.4000/studifrancesi.21526

ISSN: 2421-5856

\section{Editore}

Rosenberg \& Sellier

\section{Edizione cartacea}

Data di pubblicazione: 1 décembre 2019

Paginazione: 597

ISSN: 0039-2944

\section{Notizia bibliografica digitale}

Maria Emanuela Raffi, «Myriam Kohnen, Mobilis in mobili. Le Corps en mouvement dans la littérature du xIXe siècle», Studi Francesi [Online], 189 (LXIII | III) | 2019, online dal 01 mars 2020, consultato il 11 novembre 2021. URL: http://journals.openedition.org/studifrancesi/21526 ; DOI: https://doi.org/ 10.4000/studifrancesi.21526

Questo documento è stato generato automaticamente il 11 novembre 2021.

\section{(c) $($ ) $(9)$}

Studi Francesi è distribuita con Licenza Creative Commons Attribuzione - Non commerciale - Non opere derivate 4.0 Internazionale. 


\title{
Myriam Kohnen, Mobilis in mobili. Le Corps en mouvement dans la littérature du XIX ${ }^{\mathrm{e}}$ siècle
}

\author{
Maria Emanuela Raffi
}

\section{NOTIZIA}

Myriam Kohnen, Mobilis in mobili. Le Corps en mouvement dans la littérature du XIX ${ }^{\mathrm{e}}$ siècle, Lyon, Éditions Baudelaire, 2018, 239 pp.

1 Le mouvement, considerato come tratto distintivo della modernità del XIX secolo e posto dal titolo sotto il segno di Jules Verne, è considerato da Myriam Kohnen in tutte le declinazioni: sociale, medica, estetica, scientifica, letteraria... Corredato da alcuni Annexes costituiti da articoli di Hector Malot del 1865 sull'importanza dell'attività fisica e da un'ampia bibliografia, il volume è organizzato in tre parti - «Émotion et mouvement», "Santé et mouvement» e «Réjouissance et mouvement» -, tutte attraversate da numerosi riferimenti alla letteratura e alla presenza di specifiche attività sportive all'interno di testi di autori più o meno noti, ma il cui interesse principale si concretizza nell'evoluzione culturale di una società che si confronta con le nuove e numerose possibilità di spostamento. La passione per il canottaggio di Maupassant in Bel-Ami e En canot, descritta come ricca di implicazioni filosofiche e di riflessioni che ne fanno una sorta di "épreuve métaphysique", si affianca alla passione per la bicicletta celebrata nelle opere di vari autori, fra cui Trois villes di Zola, che ne esalta «le caractère noble et bienfaisant», fino all'automobile, «symbole de cette nouvelle vague de la rapidité» che inizia con il Novecento ed è mirabilmente rappresentata da $L a$ 628-E8 di Mirbeau. Il progressivo affermarsi del valore formativo dell'educazione fisica lungo il xIX secolo è accompagnato, nella ricognizione di Myriam Kohnen, dagli scritti di autori come Balzac, Zola, Taine e soprattutto dagli scritti di Hector Malot, per il quale «l'enseignement idéal se base [...] sur l'équilibre entre le 
corps et l'intellect», nonché di Pierre de Coubertin. Edmond de Goncourt, chiamato in causa per l'allenamento acrobatico dei protagonisti de Les Frères Zemganno e per l'ampia descrizione fisica dei due atleti, sembra invece concepire il corpo «comme une partie séparée de l'intellect» fino all'ossessione della perfezione ginnica, fonte della loro decadenza. Fondata sull'attività di "chroniqueurs" di alcuni scrittori - Zola e Malot soprattutto, ma anche Verne e Robida -, l'ultima parte dello studio è dedicata al mouvement collettivo: corse e "pédestrianisme", feste pubbliche e balli, anche se questi ultimi sono l'oggetto delle severe critiche morali di Zola. Per quest'ultimo l'A. traccia una sorta di percorso attraverso la sua opera, dal movimento come "labeur physique" (nei Rougon-Macquart) al movimento che esprime la "glorification de l'existence et la passion de l'être humain» (in Travail), molto vicino alle utopie ideate da Verne e da Robida nei loro romanzi. 